\title{
Preemptive Analgesia for Pediatric Peritoneoscopy, Comparing Caudal Block and Acetaminophen
}

\author{
STEVE GOLLADAY, M.D., SUE HUTTER, CRNA, ERIC KOEHN, RNA, \\ THURMAN HUNT, M.D., RAO GUTTA, M.D., FRANCIS GERBASI, Ph.D., \\ CYNTHIA CORPRON, M.D., AGNES HAGAN, CRNA, THOMAS DELL, CRNA, \\ and KAREN SELLEY, CRNA
}

\begin{abstract}
Background and Purpose: Although caudal block and acetaminophen have shown efficacy in postoperative pediatric pain management, they have not been compared in efficacy following peritoneoscopy.

Patients and Methods: The study population were ASA I or II children from 55 weeks postconceptual age to 10 years of age having peritoneoscopy. They were randomly assigned to preoperative caudal block using bupivacaine $0.6 \mathrm{~mL} / \mathrm{kg}$ or preoperative rectal acetaminophen $30 \mathrm{mg} / \mathrm{kg}$, with each group receiving acetaminophen $20 \mathrm{mg} / \mathrm{kg}$ per 6 hours for four doses. A blinded observer using the Objective Pain Scale assessed the children on awakening, in the PACU, and at discharge. A phone survey of satisfaction occurred on day 1. Acetaminophen was used in 18 patients, and 14 had a caudal block.

Results: The initial PACU score was $4.1 \pm 3.1$ for acetaminophen and $2.3 \pm 3.0$ for caudal block $(P=0.03)$. Fifty-six percent of the acetaminophen group needed added narcotic, whereas only $22 \%$ of those with caudal block did $(P=0.02)$. Forty three percent of the acetaminophen group had nausea compared with $11 \%$ of the caudal block group $(P=0.023)$. There was no difference in satisfaction or in parental perception of pain control at home.

Conclusion: Our results suggest that pediatric patients undergoing inguinal procedures received better pain control from caudal blocks and experienced less nausea than from highdose acetaminophen suppositories. Our results support the use of a caudal block for postoperative pain control for pediatric inguinal operations with peritoneoscopy.
\end{abstract}

\section{INTRODUCTION}

$\mathbf{P}$ Ain ASSESSMEnT AND PAIn CONTROL for the pediatric patient is difficult and often inadequate. The young nonverbal child cannot communicate pain precisely nor intelligently rate the degree of relief of pain achieved. Inadequate pain control in the pediatric patient can lead to physiological, emotional, and developmental alterations. ${ }^{1}$ Effective, safe analgesia is a high priority of every anesthesia provider for any pediatric surgical procedure. Severe unrelieved pain is one of the most common causes of unanticipated hos-

Hurley Medical Center, Flint, Michigan and Mott Children's Hospital, Ann Arbor, Michigan. 
pital admissions following what were intended to be outpatient operations. ${ }^{2}$ Rawal and associates ${ }^{2}$ surveyed patients who underwent inguinal herniorrhaphy and found that $62 \%$ experienced moderate to severe pain after discharge from the hospital. Preemptive medications have been administered to attenuate this nociceptor stimulation.

\section{PATIENTS AND METHODS}

This was a prospective single-blinded study. After Internal Review Board Approval, subjects were selected using random assignment from the surgical schedule at Hurley Medical Center. Informed consent was obtained from each subject's parent or guardian. The study population consisted of 32 pediatric patients with an ASA classification of I or II, age 55 gestational weeks to 10 years, and weight less than 33 $\mathrm{kg}$ undergoing inguinal herniorrhaphy or orchiopexy. Excluded children were those who had severe systemic disease, any absolute contraindication to regional anesthesia, a history of a neurologic condition, or a history of allergy to the study medications.

Patients arrived in the preoperative holding area 30 to 45 minutes prior to going to the operating room. Oral midazolam at a dose of $0.5 \mathrm{mg} / \mathrm{kg}$ was administered up to a maximum dose of $15 \mathrm{mg}$. Subjects were randomized into two groups. Group assignments were accomplished by random selection of sealed envelopes in groups of 20. Intraoperatively, standard monitors were used including end-tidal carbon dioxide, oxygen saturation, end-tidal gas, electrocardiography, noninvasive blood pressure, skin temperature, and peripheral nerve stimulator. Anesthesia was provided using a standardized procedure. A parent or guardian accompanied patients over the age of 12 months to the operating room for induction. Group I received an acetaminophen rectal suppository $30 \mathrm{mg} / \mathrm{kg}$ during induction of anesthesia. Group II received a caudal block administered by the pediatric surgeon who performed all the surgical procedures. As soon as practical following induction of anesthesia, the block was done using $0.25 \%$ bupivacaine at a dose of $0.6 \mathrm{~mL} / \mathrm{kg}$ up to $20 \mathrm{~mL}$ total.

A caudal block involves the injection of a local anesthetic into the sacral hiatus, a space continuous with the epidural space. With the child in a Sim's or prone position, an antimicrobial solution prepares the area, and the cornua identifies the sacral hiatus. A needle with a syringe attached begins perpendicular to the skin, advancing until the sacrococcygeal ligament is encountered. The needle is then lowered to approximately $45^{\circ}$ and advanced cephalad through the ligament. The loss of tissue resistance may be assessed with saline after which the needle is advanced parallel to the skin only a few millimeters to avoid dural or vascular puncture. ${ }^{3}$ Aspiration confirms needle placement. If no signs of vascular or dural puncture are present, the calculated dose of local anesthetic is injected slowly into the sacral canal under careful monitoring to provide anesthesia and analgesia. ${ }^{5}$ Caudal block administration time was measured from the time of patient repositioning until return to the supine position.

This report describes the experience in those children who had peritoneoscopy as part of an inguinal procedure, indirect inguinal hernia repair or orchiopexy. It did not include those children who were in a larger study that included circumcision, umbilical hernia repair, repair of a contralateral side after previous repair, or bilateral hernia repair. The caudal block group had 12 herniorrhaphies and 2 orchiopexies. The acetaminophen group had 17 herniorrhaphies and 1 orchiopexy. The peritoneoscopy was performed in all cases but one through the clinically apparent hernia side. The exception was in a boy with a nonpalpable testis who had an umbilical 2-mm endoscope used. The other children were placed in the Trendelenburg position. The peritoneoscope was introduced through the hernia sac using a $2.7-\mathrm{mm}$ port with a $0^{\circ}, 2-\mathrm{mm}$ endoscope or a 3-mm port with a $2.7-\mathrm{mm}, 30^{\circ}$ endoscope. Carbon dioxide insufflation to $6 \mathrm{~mm} \mathrm{Hg}$ was used for pneumoperitoneum. The time for pneumoperitoneum was not specifically recorded but probably averaged approximately 3 minutes. The pneumoperitoneum was evacuated through the port prior to withdrawal of the port.

The PACU nurses, the investigator who collected the pain assessment data, and the ambulatory surgical unit nurses were blinded to the type of analgesic the patient received. The blinded investigator assessed pain using the Objective Pain Scale (OPS) on patient awakening in the PACU, on discharge from the PACU, and on discharge from the ambulatory surgical unit (Table 1). The PACU personnel considered rescue anal- 
PREEMPTIVE ANALGESIA FOR PERITONEOSCOPY

Table 1. Pediatric Objective Pain Scale

\begin{tabular}{llll}
\hline Score & \multicolumn{1}{c}{0} & \multicolumn{1}{c}{1} & \multicolumn{1}{c}{2} \\
\hline Blood pressure & $\pm 10 \%$ preoperative & >20\% preoperative & $>30 \%$ preoperative \\
Crying & Not crying & Crying but responds & Crying and not \\
& & to TLC & responding to TLC \\
Movement & None & Restless & Thrashing \\
Anxiety & Asleep or calm & Mild & Hysterical \\
Posture & No special posture & Flexing legs and thighs & Holding operative site \\
\hline
\end{tabular}

gesia for those patients with a total score of $>5$. The PACU personnel initiated nonpharmacologic measures including TLC (tender loving care), parental or caregiver presence at the bedside, swaddling and holding the patient, and offering the patient oral fluid. If these measures proved unsuccessful in reducing the OPS, rescue opioid therapy of morphine sulfate up to $0.1 \mathrm{mg} / \mathrm{kg}$ was given intravenously in three doses every 5 minutes. Patients remained in PACU until standard discharge criteria were achieved. Transfer to the ambulatory surgical unit then followed.

Postoperatively, patients were assessed for their blood pressure, crying status, movement, anxiety level, and posturing (OPS score) as described above. Patient findings were assigned a numerical value to determine their total OPS score signifying their level of pain. Total OPS scores of 5 or greater indicated the need for additional pharmacologic intervention.

The child remained in the ambulatory surgical unit a minimum of 1 hour or until he or she met standard discharge criteria. Before discharge, all caregivers were educated regarding the postoperative pain control regimen. Oral acetaminophen $20 \mathrm{mg} / \mathrm{kg}$ was given every 6 hours with the times and doses specifically stated on a written take-home form. A follow-up phone call was made at 24 hours to the parent or guardian to assess the level of pain control and satisfaction with pain control on a scale of 1 to $10(1=$ least satisfied or least amount of pain control and $10=$ greatest satisfaction and greatest amount of pain control). If either score was less than 7, additional questioning ensued to determine the reason for the low score.

Of the 32 patients enrolled: 18 received acetaminophen suppositories, and 14 received caudal blocks. Statistical significance of compared data is a $P$ value of $<0.05$. Student's $t$-test was used to compare patient age, weight, preoperative vital signs, midazolam dosage, operating room time, and maximum amount of sevoflurane required to maintain anesthesia during the procedures. Chi-square analysis was used to compare postoperative nausea and vomiting, rescue analgesia intervention, type of procedure, muscle relaxant used, pain scores, and parental satisfaction scores.

\section{RESULTS}

Demographic and preoperative physiological data comparison showed no significant difference between the two groups in types of procedures, weight, age, sex, ASA classification, or preoperative vital signs. The mean time needed for administration of the caudal block was 2.6 minutes, with a range of 1 to 5 minutes. There was no significant difference between the length of operating room time or the type of analgesic method used. Group I operating room times ranged from 34 to 90 minutes with a mean of $61.4 \pm 16.3$ minutes, and Group II operating room times ranged from 20 to 119 minutes with a mean of $63.6 \pm 25.5$ minutes. The maximum amount of sevoflurane used for maintenance of anesthesia during the case was $2.75 \%$ for the caudal group and $3.1 \%$ for the acetaminophen group, showing no statistical significance. Group I had a mean PACU time of 42.5 minutes. Group II had a mean PACU time of 41.3 minutes. Group I had a mean ambulatory surgical unit time of 102.9 minutes. Group II had a mean ambulatory surgical unit time of 97.4 minutes. The time spent in the PACU and in the ambulatory surgical unit was not statistically different.

The initial pain score was significantly greater in the acetaminophen group, being $4.1 \pm 3.1$ for the acetaminophen group compared with $2.3 \pm 3.0$ for the caudal block group $(P=0.03)$ (Table 2$)$. The acet- 
Table 2. Impact of Analgesia Method on Pain, Nausea, and Parental Satisfaction

\begin{tabular}{|c|c|c|c|}
\hline & $\begin{array}{l}\text { Acetaminophen } \\
(\mathrm{n}=18)\end{array}$ & $\begin{array}{c}\text { Caudal } \\
(\mathrm{n}=14)\end{array}$ & $\mathrm{P}$ value \\
\hline Arrival to PACU pain score & $4.1 \pm 3.1^{\mathrm{a}}$ & $2.3 \pm 3.0$ & 0.03 \\
\hline$\%$ of patients having morphine, 1 dose & 56 & 22 & 0.02 \\
\hline$\%$ of patients having morphine, 2 doses & 43 & 0 & 0.001 \\
\hline$\%$ of patients with nausea & 43 & 11 & 0.023 \\
\hline Parental satisfaction at 24 hours, linear scale $1-10$ & $8.1 \pm 2.1$ & $8.0 \pm 1.8$ & NS \\
\hline
\end{tabular}

${ }^{\mathrm{a}}$ Mean $\pm \mathrm{SD}$.

aminophen group also required significantly more postoperative pain medication than the caudal group. Fifty-six percent of the acetaminophen patients (10 of 18) required a first-time dose of morphine compared with $22 \%$ (3 of 14) of the caudal block patients $(P=0.02)$ (Table 2). Forty-three percent (8 of 18) of the acetaminophen patients received a second dose of morphine compared with none of the caudal group $(P=$ $0.001)$ (Table 2). Eleven percent ( 2 of 18$)$ of Group I patients received a third dose of morphine $(P=0.11)$, whereas none of the Group II patients required a third dose.

The mean pain score on discharge from the PACU for Group I was $1.0 \pm 1.5$ compared with $0.4 \pm 0.7$ for Group II. The mean pain score on discharge from the ambulatory surgical unit for Group I was $0.2 \pm$ 0.5 compared with $0.4 \pm 1.2$ for Group II. The mean pain score on discharge from the PACU and discharge from the ambulatory surgical unit were not statistically different. Forty-three percent of Group I (8 of 18 patients) experienced nausea compared with $14 \%$ ( 2 of 14 patients) of Group II $(P=0.023)$ (Table 2).

Twenty-four hour follow-up assessment of parental satisfaction and rating of pain control showed no significant difference between the two groups. The linear scale mean satisfaction and pain control score for Group I was $8.1 \pm 2.1$ and $8.0 \pm 1.8$, respectively, and for Group II, the scores were $8.5 \pm 1.7$ and $8.6 \pm$ 1.3 (Table 2). The 6-hour oral acetaminophen dose was given to $90 \%$ of the patients, the 12-hour dose was given to $81 \%$ of the patients, and the 18 -hour dose was given to $87 \%$ of the patients. Parental satisfaction and rating of pain control showed that nine parents had a score of 7 or lower out of a possible 10. Of these nine patients, two did not receive the 6-hour dose, two did not receive the 12-hour dose, and three did not receive the 18-hour dose. The statistical comparison of parental satisfaction and pain control score of 7 or lower with the patients not receiving the 18-hour acetaminophen dose showed significance $(P=0.04)$.

Cost analysis indicates caudal blocks cost approximately $\$ 68.64$ (operating room time, two injection needles, one syringe, sterile gloves, and bupivacaine) and additional operating room and anesthetic time, which is small enough not affect the total. Acetaminophen suppository administration costs approximately $\$ 0.22$ (two nonsterile gloves and suppository).

\section{DISCUSSION}

The purpose of this study was to investigate the efficacy of an acetaminophen suppository $30 \mathrm{mg} / \mathrm{kg}$ and $0.25 \%$ bupivacaine caudal block $0.6 \mathrm{~mL} / \mathrm{kg}$ in controlling pediatric postoperative pain in inguinal procedures including peritoneoscopy as well as a determination of parental satisfaction. Various methods of analgesia used for pain control after pediatric surgical procedures include narcotics, nonsteroidal anti-inflammatory drugs (NSAIDs), infiltration of local anesthetic at the operative site, use of a regional anesthetic block, and acetaminophen. ${ }^{3}$ Each of these methods possesses variable success, and each has potential problems. ${ }^{3}$ Infiltration of local anesthetic or regional block usually offers effective and immediate pain control locally and systemically, but after several hours, pain ensues. Caudal blocks, although highly effective in providing intraoperative and postoperative analgesia, have potential complications, namely hematoma, improper injection of the local anesthetic (either subcutaneously or intrathecally, with resultant ineffective block), infection, or systemic toxicity caused by the intravascular injection of local anesthetic. ${ }^{4}$ The literature has many references to the simplicity of performance, reliability, and safety for infants and children. ${ }^{4-7}$ 


\section{PREEMPTIVE ANALGESIA FOR PERITONEOSCOPY}

A study by Ho and associates ${ }^{8}$ compared the timing of the caudal block, either preoperative or postoperative before emergence, and found no difference in the postoperative analgesic requirements in the two groups. Narcotics have side effects such as nausea, vomiting, excessive sedation, and resultant respiratory compromise. Nonsteroidal anti-inflammatory drugs are associated with an increased risk of bleeding because of their effect on platelet aggregation and can cause gastrointestinal distress. Acetaminophen inhibits the synthesis of prostaglandin in the central nervous system and peripherally blocks pain impulse generation by blocking the activation of nociceptors. Acetaminophen has the problem of reproducibility attaining a therapeutic level, as proper dose and timing may be difficult to achieve. The therapeutic serum concentration is 10 to $30 \mu \mathrm{cg} / \mathrm{mL}$, with the toxic concentration considered to be greater than $200 \mu \mathrm{g} / \mathrm{mL}$.

Rectal dosing of $20 \mathrm{mg} / \mathrm{kg}$ has resulted in subtherapeutic plasma concentrations, but therapeutic plasma concentrations could be achieved with rectal doses of 35 to $45 \mathrm{mg} / \mathrm{kg} .{ }^{10-12}$ Birmingham and colleagues ${ }^{12}$ suggest loading doses of rectal acetaminophen of $40 \mathrm{mg} / \mathrm{kg}$ followed by subsequent doses of $20 \mathrm{mg} / \mathrm{kg}$ every 4 to 6 hours to produce therapeutic blood levels of 10 to $20 \mu \mathrm{g} / \mathrm{mL}$ during the first 24 hours postoperatively. Children benefit from scheduled administration of postoperative analgesics, as sporadic administration leads to peaks and troughs in blood levels of the drug, allowing pain to intensify between doses. ${ }^{13}$ Anderson and Holford ${ }^{14}$ used a computer simulation model to predict rectal acetaminophen dosing schedules that would maintain steady-state plasma concentrations of 10 to $20 \mathrm{mg} / \mathrm{L}$. This concentration can be reached after a loading dose of $70 \mathrm{mg} / \mathrm{kg}$ and a maintenance dose of $50 \mathrm{mg} / \mathrm{kg}$ every 8 hours. ${ }^{14}$

Hannallah's Objective Pain Scale includes five areas of observation: blood pressure, anxiety, movement, crying, and posture ${ }^{15}$ (see Table 1). This scale has shown reliability and validity in assessing postoperative pain in children. When compared with the Children's Hospital of Eastern Ontario Pain Scale, the OPS demonstrates concurrent validity. Researchers have concluded that Hannallah's scale is a valid tool and can be used to assess pain in the pediatric population. ${ }^{15}$

Our results suggest that pediatric patients having inguinal surgical procedures receive better pain control and experienced less nausea from caudal blocks than from high-dose acetaminophen suppositories. The caudal block patients exhibited lower pain scores on admission to the PACU, requiring less pharmacologic intervention. Fifty-six percent of the acetaminophen patients required a first-time dose of intravenous morphine for postoperative pain control, compared with $22 \%$ of the caudal block group. Forty-three percent of the acetaminophen group and none of the caudal group required a second dose of intravenous morphine. Also, $13 \%$ of the acetaminophen group but none of the caudal block group required a third dose of morphine. Forty-three percent of the acetaminophen group experienced nausea compared with $11 \%$ of the caudal group $(P=0.023)$. The difference in pain control may relate to peak concentration of analgesic medications. The time to peak serum concentrations of an acetaminophen suppository is approximately 100 minutes. ${ }^{16}$ The surgical procedures had a mean operating room time of 61.4 minutes, possibly not allowing the acetaminophen time to reach optimal analgesic levels. The rectal absorption of acetaminophen has been shown to be erratic and incomplete in previous studies, resulting in less than therapeutic serum levels required for analgesia. ${ }^{16,17}$ The $20 \mathrm{mg} / \mathrm{kg}$ of 6 hours oral acetaminophen provided a 24-hour linear pain scale postoperative pain score of $8.0 \pm 1.8$ for Group I and 8.6 \pm 1.3 for Group II (scale 1-10), suggesting adequate postoperative pain control.

Azurin and associates ${ }^{18}$ from the Department of Surgery, Pennsylvania Hospital, did a prospective study on the efficacy of epidural anesthesia for endoscopic preperitoneal herniorrhaphy. There was only one conversion to general anesthesia. ${ }^{18}$ Bridenbaugh and Soderstrom ${ }^{19}$ studied lumbar epidural block anesthesia for outpatient laparoscopic tubal sterilization. Oxygenation during the period of pneumoperitoneum was adequate. The technique resulted in a shortened postanesthesia recovery period and fewer postanesthesia complications. ${ }^{19}$ Caudal epidural block was more effective than local infiltration in controlling pain and allowed early discharge of children after inguinal operations at Vanderbilt. ${ }^{20}$ It is therefore clear that a caudal block controls most of the pain associated with the procedure and with the accompanying pneumoperitoneum. Although a caudal block can achieve as high as a sensory level, levels below that are more common. The lower levels would not be expected to limit shoulder pain. The absence of expressed shoulder pain in the group may relate to the low pressure $(6 \mathrm{~mm} \mathrm{Hg})$, the short duration of the pneumoperitoneum (about $3 \mathrm{~min}$ utes), maintenance of Trendelenburg position during the pneumoperitoneum, nearly complete evacuation 
of the pneumoperitoneum, or the inability of a young child to localize pain. Regardless of the factor or factors responsible, the results of our study show superiority of caudal block to acetaminophen.

Recovery room nurses have preconceived ideas about how effective an acetaminophen suppository may be. Nurses in the PACU were challenged to keep their verbal prejudice from the parents, who are easily swayed by comments such as "he must have only had the suppository" or "I'll get the morphine ready" rather than encourage TLC measures. Verbal pediatric patients may complain of pain even though they are exhibiting none of the signs and symptoms of postoperative pain. Postanesthesia care unit nurses have been observed to administer rescue analgesia rather than explore the level or the location of pain. One child was screaming that his back hurt. His mother and the nurse both reacted to the child by demanding morphine. The investigator was able to determine that the child was complaining that his shoulders were hot from the intraoperative warming blanket, and simply placing an icebag on the affected area reduced the child to a calm, comfortable, sleepy state. At other times, nursing staff were convinced that a patient had received a caudal block because of the complete level of comfort only to find out later that the patient had received the acetaminophen suppository.

Nausea was more prevalent in the acetaminophen group, perhaps related to the analgesic. Nausea in pediatric patients can be attributed to many sources. Forty-three percent of the acetaminophen group experienced nausea compared with $11 \%$ of the caudal group $(P=0.023)$. Five of seventeen patients who required additional postoperative pain medication exhibited postoperative nausea or vomiting, whereas none who did not receive morphine did so $(P=0.004)$. It would appear that morphine contributed to the episodes of nausea and vomiting through stimulation of the receptors in the chemotrigger zone.

Suggested follow-up research includes delivery of acetaminophen, perhaps at a higher dose, earlier in the preoperative period, allowing the medication to reach its peak plasma concentration before the initiation of surgery. The patient would then benefit from the effects of acetaminophen inhibiting the synthesis of prostaglandins in the central nervous system and peripherally blocking the pain impulse generation.

\section{CONCLUSION}

The acetaminophen suppository appears to be more cost effective. However, other factors that may increase cost must be considered such as increased use of anesthetic agents, later discharge time, and greater need for pharmacologic interventions to treat nausea and excessive pain. Institutions and individuals must compare the total costs of each method as well as their effectiveness before endorsing one method over another. Our results support the use of caudal blocks for postoperative pain control of pediatric inguinal surgical procedures.

\section{REFERENCES}

1. Eland J. Pain in children. Nurs Clin North Am 1990;25:871-884.

2. Rawal N, Hylander J, Nydahl P, Olofsson I, Gupta A. Survey of postoperative analgesia following ambulatory surgery. Acta Anaesthesiol Scand 1997;41:1017-1022.

3. Tobias J. Postoperative pain management. Pediatr Ann 1997;26:490-500.

4. Morgan G, Mikhail M. Clinical Anesthesiology. Stanford, CT: Appleton \& Lange, 1996, pp 196-199, $240-242$.

5. Gregory G. Pediatric Anesthesia. New York: Churchill-Livingstone, 1994, pp 290-294.

6. Catterall W, Mackie K. Local anesthetics. In: Hardman JG, Limbird LL, Molinoff PB, Ruddan RW, Gilman AG (eds): Goodman \& Gilman's The Pharmacologic Basis of Therapeutics, ed 9. New York: McGraw Hill, 1996, pp 339,345 .

7. Barash P, Cullen B, Stoelting R. Clinical Anesthesia, ed 3. New York: Lippincott-Raven, 1997, pp 829, 1343.

8. Ho J, Khambatta H, Pang L, Siegfried R, Sun L. Preemptive analgesia in children: Does it exist? Regional Anesth 1997;22:125-130. 


\section{PREEMPTIVE ANALGESIA FOR PERITONEOSCOPY}

9. Donnelly A, Cunningham F, Baughman V. Anesthesiology and Critical Care Drug Handbook. Cleveland: LexiComp, 1999, pp 20-23, 135-137, 372-376, 433-435, 624-626, 632-635, 640-643, 683-684, 867.

10. Montgomery C, McCormach J, Reichert C, Marsland C. Plasma concentration after high dose rectal acetaminophen in children. Can J Anesth 1995;42:982-986.

11. Houck C, Sullivan L, Wilder K, Rusy L, Burrows F. Pharmacokinetics of a higher dose of rectal acetaminophen in children. Anesthesiology 1995;83(3A):1126.

12. Birmingham P, Tobin M, Henthorn T, Kennedy-Mooney S, Smith F, Cote C. Loading and subsequent dosing of rectal acetaminophen in children: A 24 hour pharmacokinetic study of new dose recommendations. Anesthesiology 1996;85(3A): 1105.

13. Hopkins C, Underhill S, Booker P. Pharmacokinetics of paracetamol after cardiac surgery. Arch Dis Childhood 1990;65:971-976.

14. Anderson B, Holford N. Rectal paracetamol dosing regimens: Determination by computer simulation. Paediatr Anaesth 1997;7:451-455.

15. Noreen J, Hannallah R, Getson P, O’Donnel R, Kelliher G, Walker N. Concurrent validation of an objective pain scale for infants and children. Anesthesiology 1991;75(3A):A934.

16. Romej M, Voepel-Lewis T, Merkel S, Reynolds P, Quinn P. Effect of preemptive acetaminophen on postoperative pain scores and oral fluid intake in pediatric tonsillectomy patients. J Am Assoc Nurse Anesthetists 1996; 64:535-540.

17. Gaudreault P, Guay J, Nicol O, Duphis C. Pharmacokinetics and clinical efficacy of intrarectal solution of acetaminophen. Can J Anaesth 1988;35:149-152.

18. Azurin DJ, Go LS, Cwik JC, Schuricht AL. The efficacy of epidural anesthesia for endoscopic preperitonealherniorrhaphy: A prospective study. J Laparoendosc Surg 1996;6:369-373.

19. Bridenbaugh LD, Soderstrom RM. Lumbar epidural block anesthesia for outpatient laparoscopy. J Reprod Med $1979 ; 23: 85-86$.

20. Tobias JD, Holcomb GW 3rd, Brock JW 3rd, Morgan WM 3rd, O’Dell N, Lowe S, Rasmussen GE. Analgesia after inguinal herniorrhaphy with laparoscopic inspection of the peritoneum in children: Caudal block versus ilioinguinal/iliohypogastric block. Am J Anesth 1995;22:193-197.

Address reprint requests to: Eustace Stevers Golladay, M.D. Division of Pediatric Surgery Mott Children's Hospital F-3970

1500 E. Medical Center Drive Ann Arbor, MI 48109

E-mail: Egoll@umich.edu 
This article has been cited by:

1. Jyoti Borkar , Nandini Dave . 2005. Analgesic Efficacy of Caudal Block Versus Diclofenac Suppository and Local Anesthetic Infiltration Following Pediatric LaparoscopyAnalgesic Efficacy of Caudal Block Versus Diclofenac Suppository and Local Anesthetic Infiltration Following Pediatric Laparoscopy. Journal of Laparoendoscopic Advanced Surgical Techniques 15:4, 415-418. [Abstract] [PDF] [PDF Plus] 